\title{
La vanne de réglage Monovar
}

\author{
A. Bonazzi Alsthom-ACB Centre d'études \& de recherches de Grenoble (CERG)
}

T. Fournier Alsthom-Fluides La Courneuve

Le Monovar est une vanne de réglage multijets réalisant une dissipation d'énergie répartie au sein du fluide et minimisant ainsi les perturbations à l'aval. Créé pour répondre à des besoins de recherche en matière de cavitation, le Monovar présente un excellent coefficient de début de cavitation, ainsi qu'une faible sensibilité à l'érosion de cavitation. Ses performances dans ce domaine ont fait l'objet de nombreux essais.

\section{Historique}

L'historique du Monovar est étroitement lié aux recherches menées en France sur les phénomènes de cavitation. En effet, lorsque la Sogreah décida de construire un tunnel de cavitation, le problème se posa de monter à l'aval de la section d'essais un dissipateur d'énergie aussi silencieux que possible. Aucune vanne conventionnelle ne permettait de répondre à ce problème.

Les plaques perforées étant utilisées depuis longtemps comme dissipateurs d'énergie ou comme débitmètres, il fut décidé de monter deux plaques perforées identiques, comme indiqué sur la figure 1 .

La vanne était ouverte lorsque les orifices coïncidaient ; elle était fermée lorsque les orifices d'une plaque étaient face à une zone non perforée de l'autre.

Ce dispositif se révéla très performant : aucune perturbation à basse fréquence de l'écoulement n'était créée, et le système présentait très peu de cavitation, même aux faibles valeurs de sigma.

1. Première vanne Monovar montée sur un tunnel de cavitation.

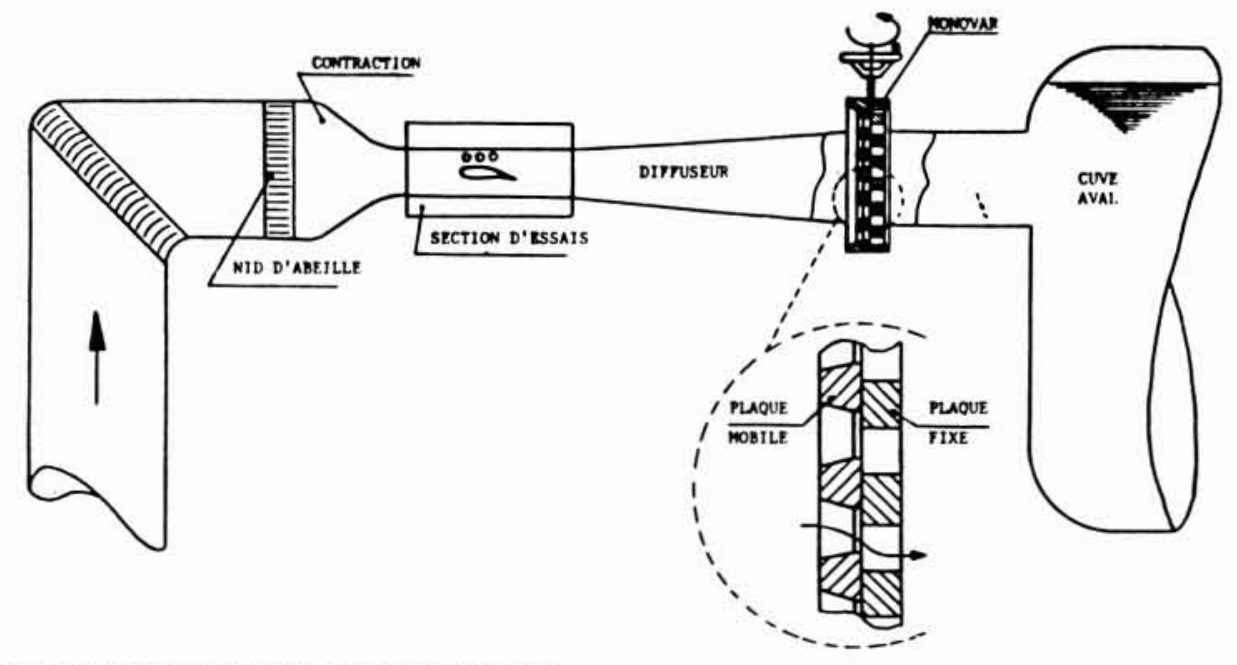

The Monovar adjustment valve.

The Monovar is a multijet adjustment valve dissipating energy inside the fluid and thus minimizing the downstream disturbances. Designed so as to respond to research needs in the field of cavitation, the Monovar offers a sound coefficient of initial cavitation, as well as a low sensitivity to cavitation erosion. Its true performances in this field have given rise to many tests. 

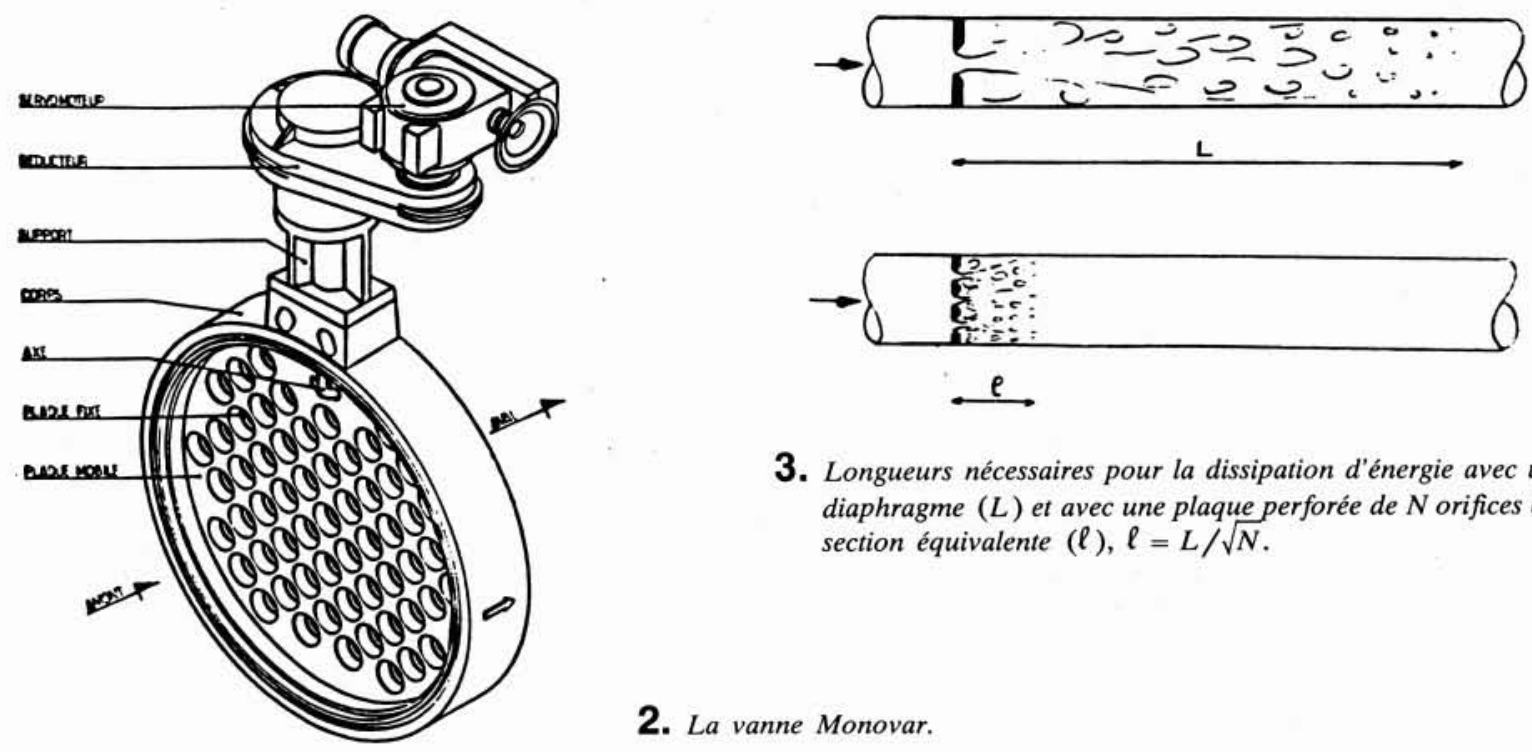

3. Longueurs nécessaires pour la dissipation d'énergie avec un diaphragme $(L)$ et avec une plaque perforée de $N$ orifices de section équivalente $(\ell), \ell=L / \sqrt{N}$.

2. La vanne Monovar.

En tant que fabricant de vannes, Neyrtec, puis Alsthom-Fluides ont développé une version commerciale de ce dispositif (Cf. fig. 2), initialement pour des applications en réseaux de distribution d'eau (adduction, irrigation), puis dans d'autres domaines industriels, notamment l'énergie nucléaire et pétrolière.

La vanne Monovar était née, et bien que de nombreuses améliorations technologiques aient été apportées pour répondre à des applications de plus en plus nombreuses, ses principes de base sont restés inchangés.

\section{Les avantages du Monovar}

La figure 3 montre comment la dissipation d'énergie se produit en aval d'un diaphragme ou en aval d'une plaque perforée.

Dans le premier cas, une longueur de conduite d'environ dix fois son diamètre est nécessaire.

Dans le cas d'une plaque perforée, la dimension caractéristique n'est plus le diamètre de la conduite, mais celui d'un orifice. De ce fait, par rapport à un diaphragme dissipant l'énergie sur une longueur $L$, une plaque perforée de même section de passage comportant $N$ orifices dissipe l'énergie sur une longueur $L / \sqrt{N}$.

Si la plaque comporte 57 orifices, comme le Monovar standard, la longueur nécessaire à la dissipation d'énergie est d'environ 1,3 diamètre de tuyauterie.

Les avantages d'une longueur de dissipation aussi faible sont évidents :

- pas d'interaction avec les organes placés en aval,

- niveau de bruit réduit,

- érosion de cavitation réduite du fait que la dimension caractéristique de chaque jet est plus faible et qu'ils sont éloignés de la paroi (dissipation au sein du fluide),

- distribution de vitesse améliorée à l'aval,

- possibilité d'utilisation de la vanne comme organe déprimogène pour la mesure du débit.

\section{Monovar et cavitation}

\subsection{Introduction}

Les utilisateurs de la vanne Monovar peuvent être classés en trois catégories en fonction du service qu'ils attendent :

- régulation de débit avec loi ouverture-débit connue et fidèle,

- réglage de débit avec des niveaux de bruit, de vibrations et de fluctuations de pression réduits,

- réglage ou régulation de débit en absence de toute cavitation dans et à l'aval de la vanne.

Les utilisateurs de la première catégorie peuvent admettre de la cavitation dans et à l'aval de la vanne, à condition que celle-ci n'influe pas sur les caractéristiques de débit de la vanne et, bien entendu, qu'elle ne provoque pas d'érosion de la vanne ou de la tuyauterie.

Ceux de la seconde catégorie admettent une cavitation de début, ou même légèrement développée, mais ne provoquant pas de bruit et de vibrations et fluctuations de pression trop importantes.

Pour les Monovar installés par exemple sur des boucles d'essais de laboratoire, les utilisateurs de la troisième catégorie n'admettent pas la moindre cavitation dans et à l'aval de la vanne.

Pour répondre correctement aux besoins de ces différents utilisateurs, il était nécessaire de bien connaître les caractéristiques de cavitation des Monovar.

Dans cette optique ont été conduits des essais au Centre d'études \& de recherches de Grenoble (CERG) d'Alsthom-ACB, en faisant varier le paramètre sigma caractéristique de la cavitation, et dont nous rappelons ci-après la définition :

$$
\sigma=\frac{P_{\text {aval }}-P_{\text {vapeur }}}{\Delta P}
$$




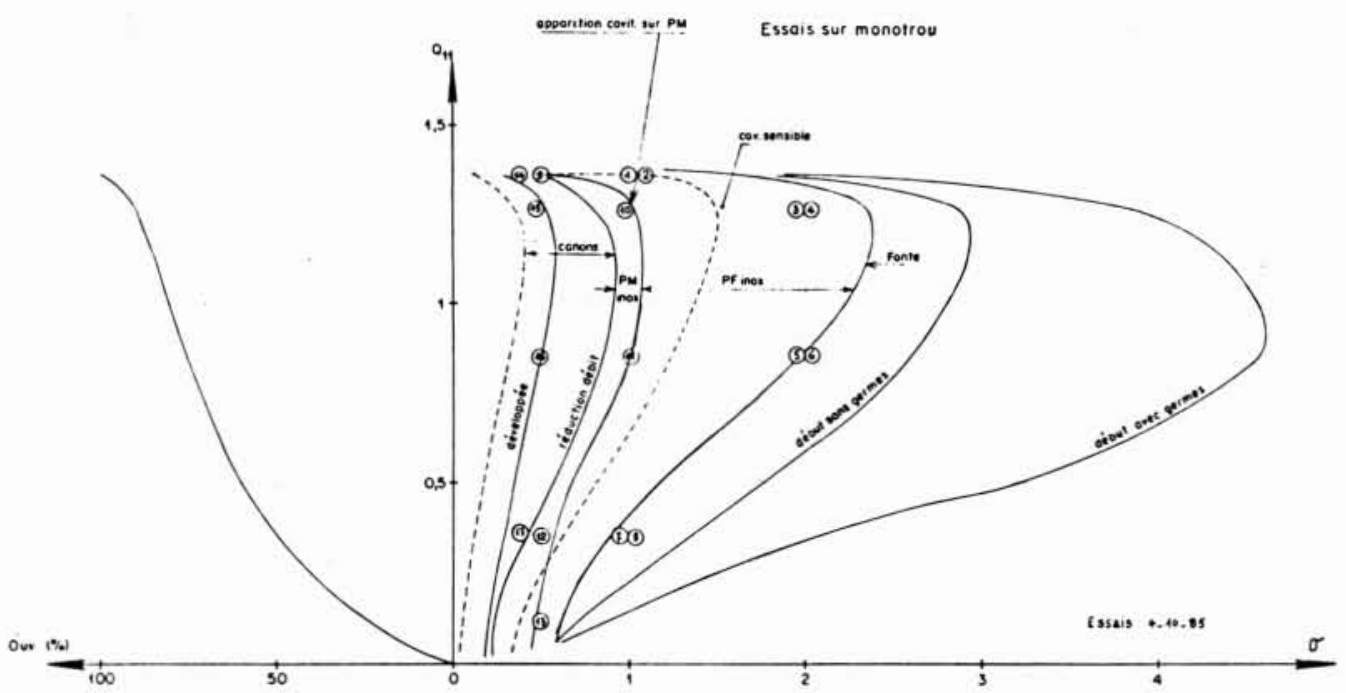

4. Cavitation: Monovar.

où $P_{\text {aval }}$ est la pression statique absolue à l'aval de la vanne, $\Delta P$ la perte de pression dans la vanne et $P_{\text {vapeur }}$ la tension de vapeur du fluide.

Les essais ont permis de déterminer les conditions de début de cavitation (éclatement de la première bulle de vapeur), celles correspondant à une cavitation caractérisée, puis celles provoquant une modification de la débitance de la vanne.

\subsection{Visualisation des figures de cavitation d'un Monovar}

Les essais de visualisation ont été conduits sur un modèle monotrou placé dans un tunnel d'essais à grande vitesse. Le modèle représentait une perforation d'un Monovar DN 500 PN 25 de la gamme standard.

\section{Mesures effectuées}

- position de la plaque mobile,

- débit à travers le monotrou,

- mesure des pressions amont et aval. La pression aval était prise juste après la plaque fixe et à une distance de quatre diamètres de tuyauterie aval,

- photographie des différentes figures de cavitation.

\section{Similitude}

La similitude à appliquer est la similitude de Thoma qui impose de respecter le paramètre de cavitation sigma dont la définition est rappelée au $\$ 3.1$ ci-dessus.

Pour une même valeur du paramètre sigma sur le modèle et sur le réel, on observe la même figure de cavitation. Ceci est vrai dans notre expérience pour ce qui se passe dans le monotrou et dans un trou du Monovar réel. Le modèle monotrou ne peut évidemment pas représenter ce qui se passe à l'aval du Monovar réel et notamment l'interaction possible entre les jets multiples issus des 57 trous de la plaque fixe. Le fait que la cavitation observée, tant au début que lorsqu'elle est bien développée, soit pratiquement confinée dans le trou du Monovar justifie la validité du modèle monotrou en tant que représentant de la vanne réelle.

\section{Essais réalisés}

Le monotrou a été successivement positionné aux ouvertures $100 \%, 90 \%, 75 \%, 50 \%$ et $25 \%$. Pour chaque valeur de l'ouverture, on a réglé le $\Delta P$ aux bornes du monotrou à 1 bar puis on a cherché par réglage du niveau général de pression dans le moyen d'essai les figures de cavitation suivantes :

- début de cavitation, c'est-à-dire la toute première apparition de petites bulles de vapeur détectées visuellement et/ou au bruit de collapse par l'opérateur. Ce début correspond typiquement à cinq ou six collapses par minute ;

- cavitation caractérisée qui correspond à l'apparition continue de bulles ou petites poches de vapeur et à un bruit de collapse permanent ;

- cavitation importante conduisant à une chute de caractéristiques de la vanne (réduction de débit pour un même $\Delta P$ aux bornes ;

- cavitation très développée.

La détermination des débuts de cavitation a été effectuée avec deux qualités d'eau :

- eau avec très faible teneur en air dissous et très petit nombre de germes de cavitation correspondant aux conditions de certains essais en laboratoire d'hydraulique ;

- eau saturée en air dissous à pression atmosphérique et contenant une quantité de germes de cavitation correspondant aux conditions des circuits industriels classiques.

\section{Résultats}

Les résultats obtenus sont donnés sur la figure 4. On a représenté l'évolution des sigmas en fonction du débit (donc de l'ouverture) pour les cavitations de début, caractérisées, à réduction de débit et développées. 


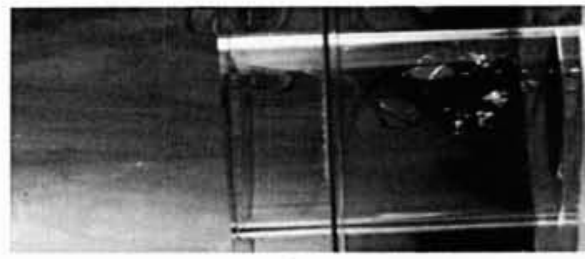

ouv. $=75 \%$

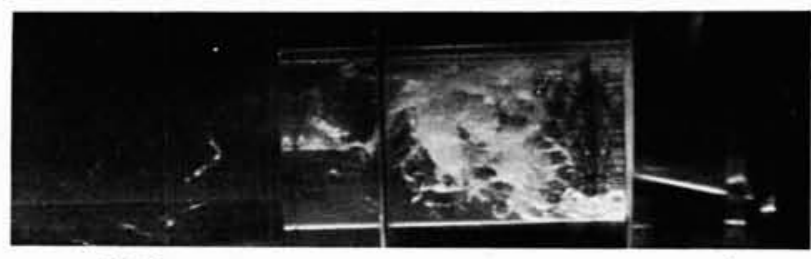

ouv. $=75 \%$

$\sigma=1$
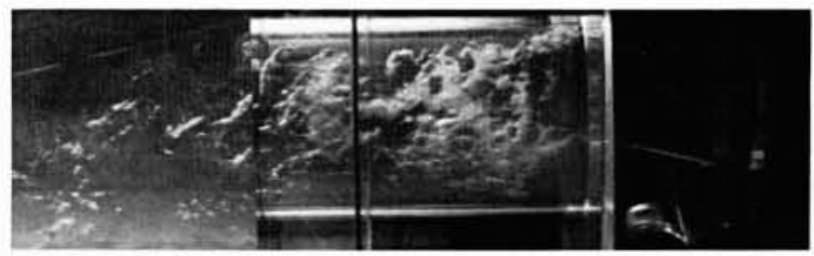

ouv. $=50 \%$

$\sigma=0,5$

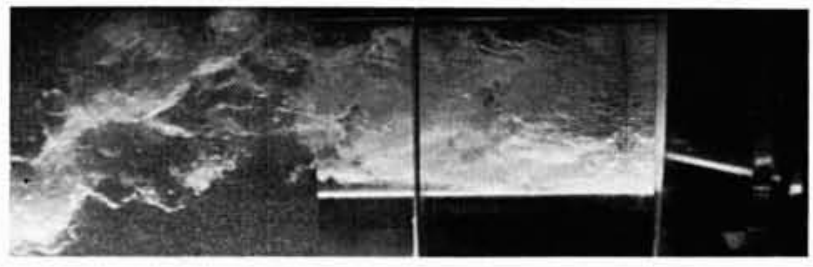

ouv. $=75 \%$

$$
\sigma=0,5
$$

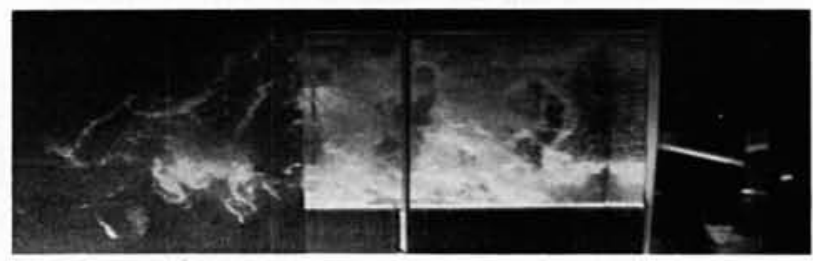

ouv. $=50 \%$

$\sigma=0,4$

5. Cavitation: Monovar.

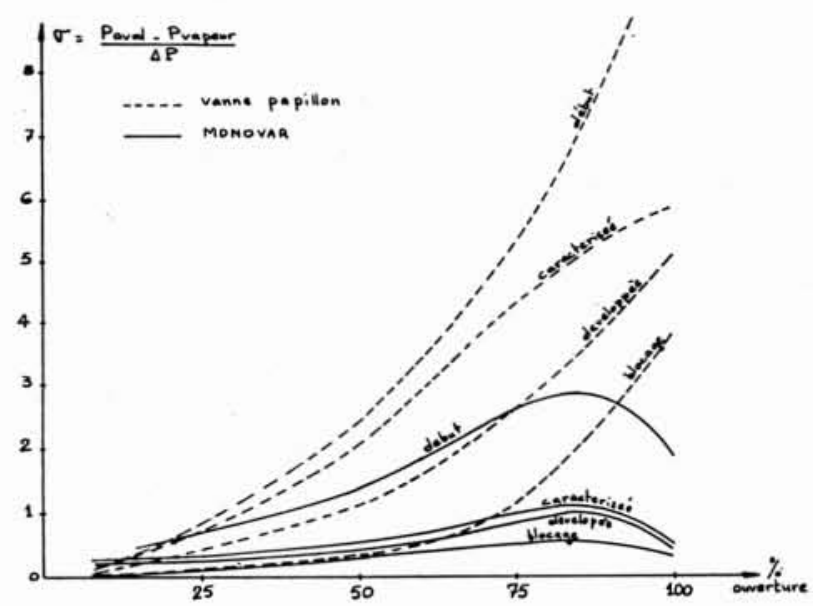

6. Cavitation : vanne papillon.

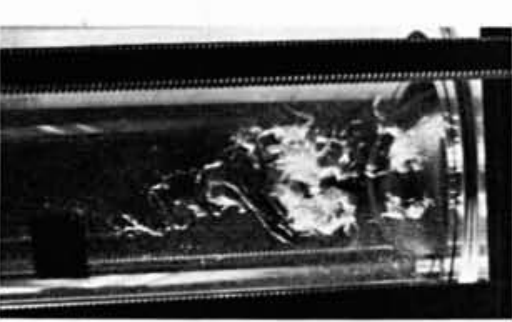

ouv. $=56 \%$

$\sigma=1,4$
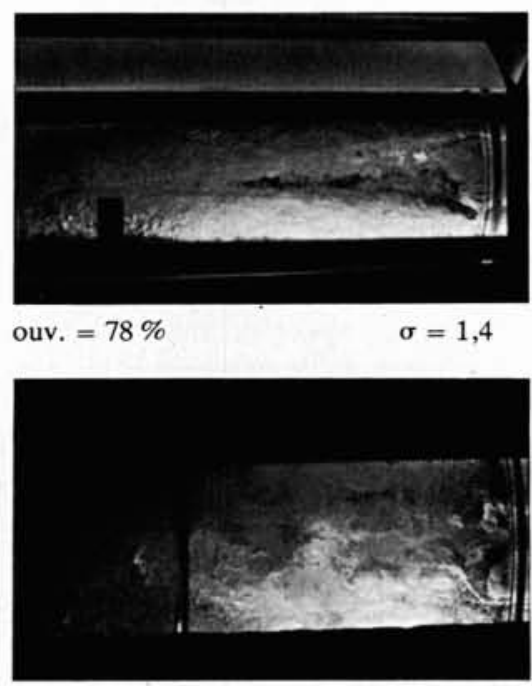

ouv $=56 \%$

$\sigma=0,5$

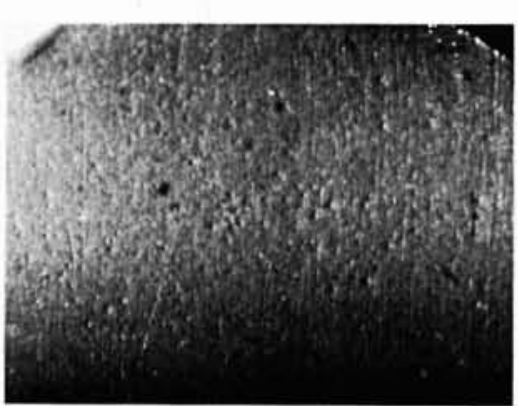

Aval papillon

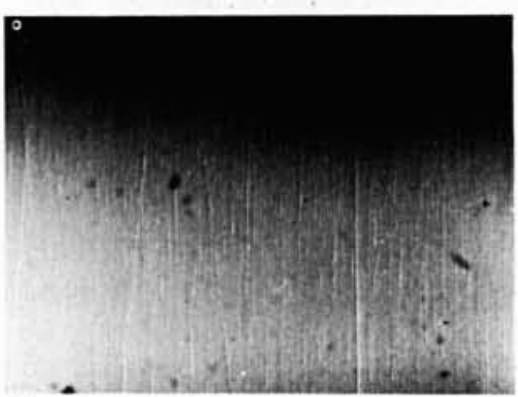

Plaque aval Monovar

7. Plaque aval Monovar. Essais d'érosion. Temps $=1$ heure, matériau: aluminium, ouverture $44 \% \Delta P=1,8 \mathrm{bar}$ $\sigma=0,24$. 
On remarque que l'ouverture de vanne la plus critique se situe vers $85 \%$. Les photographies des diverses figures de cavitation sont repérées sur la figure 4 et données figures 5 et 6.

A titre de comparaison, on donne les photographies de figures de cavitation d'une vanne papillon classique à des sigmas et ouvertures similaires.

\subsection{Comparaison sommaire des érosions par cavitation dans un Monovar et à l'aval d'une vanne papillon}

Des éprouvettes d'aluminium pur ont été disposées d'une part à l'aval d'une vanne papillon et d'autre part dans le trou de la plaque fixe du Monovar.

Les conditions d'essais sont les suivantes:

- ouverture des vannes : $44 \%$

$$
\begin{array}{ll}
-\Delta P \text { aux bornes } & : 1,78 \text { bar } \\
-\sigma=0,24 & : 1 \mathrm{~h} .
\end{array}
$$

Résultats: Sur les quatre éprouvettes disposées à l'aval du papillon, deux présentaient des traces d'érosion et deux étaient intactes. L'ensemble de la surface interne du trou de la plaque fixe du Monovar était intacte.

Les photos (fig. 7) de l'éprouvette montrent une éprouvette érodée à l'aval du papillon et la surface du trou du Monovar.

\subsection{Comparaison avec le Monovar et une vanne-papillon}

Dans le cadre d'une étude particulière, notre laboratoire a réalisé des essais de visualisation des figures de cavitation d'une vanne-papillon de type classique. Ces essais ont été conduits de façon identique à ceux réalisés pour le Monovar et par les mêmes opérateurs. Les résultats obtenus sont donc directement comparables. La figure 8 montre l'ensemble des résultats obtenus.

Il est net que le Monovar est bien meilleur tant en début de cavitation, ce qui est intéressant chaque fois que les problèmes de bruit sont importants, que pour les cavitations développées et de blocage de débit.

Pour ce dernier cas, on voit que la débitance naturelle du Monovar qui est plus faible que celle d'un papillon de même diamètre, peut devenir équivalente dès lors que l'on fonctionne avec une forte cavitation et à des ouvertures supérieures à $75 \%$.

\section{Exemples d'utilisation du Monovar}

\subsection{Utilisations en laboratoire}

Dans certaines applications de laboratoire, des charges importantes doivent être dissipées sans aucune cavitation.

La seule solution est d'installer des vannes en série, chacune ayant un sigma de début de cavitation $\sigma_{i}$ aussi bas que possible. Le nombre minimal de vannes à installer est donné par :

$$
n=\log \left[\left(\sigma_{i g}+1\right) / \sigma_{i g}\right] / \log \left[\left(\sigma_{i}+1\right) / \sigma_{i}\right]
$$

où $\sigma_{i g}$ est le sigma d'installation du système complet.
Pour minimiser le nombre de vannes, il faut que leur $\sigma_{i}$ soit aussi bas que possible. Le Monovar se prête bien à cette application, car d'une part il a l'un des meilleurs $\sigma_{i}$ des vannes de régulation, et d'autre part il a un encombrement très faible qui facilite le montage en série.

Le Monovar équipe de nombreux centre d'essais de matériel hydraulique, notamment: EDF (boucle EPOCA à Chatou), DCAN Indret (boucle d'essai de pompes), ENSAM Lille (boucle d'essai de pompes), KSB (boucle d'essai de pompes nucléaires), CREMHYG (boucle d'essai de pompes et turbines), Alsthom-ACB CERG (tunnel à haute vitesse).

\subsection{Utilisations industrielles}

Dans la plupart des utilisations industrielles classiques, le problème n'est pas d'éviter toute cavitation, mais de travailler dans un domaine où la cavitation éventuelle ne provoque ni perturbation d'écoulement, ni érosion.

Les essais réalisés au CERG ont permis de situer le sigma limite à partir duquel se produit une réduction de débit à ouverture donnée.

Les phénomènes d'érosion de cavitation sont plus difficiles à prévoir et quantifier. En se basant à la fois sur les résultats d'essais et sur son expérience de constructeur, Alsthom-Fluides préconise des matériaux adaptés au degré de cavitation :

- plaque fixe en inox au lieu de fonte pour la zone de cavitation caractérisée ;

- plaque mobile en inox lorsqu'on atteint la cavitation développée ;

- chemisage des perforations de la plaque fixe par des canons en carbure de tungstène lorsqu'on atteint la courbe de réduction de débit.

Dans certains cas, il est préconisé de monter à l'aval du Monovar un diaphragme qui dissipe une partie de la charge et peut permettre de faire fonctionner le Monovar dans une zone de cavitation moins critique.

\section{Références}

P. SChummer, Alsthom ACB/CERG. Rapport CERG R. 20740 , «Etude des caractéristiques débit/ouverture des Monovar de la gamme standard».

A. Bonazzi \& J. P. MAsson, Alsthom ACB/CERG. Rapport CERG R. 20765 , «Monovar, Etude cavitation».

Y. LECOFFrE, Ingénieur-Conseil en hydraulique, Rapport Alsthom-Fluides, "Multijet Plate Control Valve Monovar by Alsthom-Fluides », 1987.

Adresses des auteurs

$\begin{array}{ll}\text { Monsieur A. Bonazzi } & \text { Monsieur T. Fournier } \\ \text { Alsthom CERG } & \text { Alsthom-Fluides } \\ \text { Voie privée Rhône-Poulenc } & 141 \text {, rue Rateau } \\ 38800 \text { Le Pont de Claix } & 93123 \text { La Courneuve } \\ \text { Tél. : } 1676409040 & \text { Tél. : } 48389289\end{array}$


Mme NIENALTOWSKA : Dans vos essais concernant l'apparition de la cavitation, vous avez travaillé sur un orifice unique. Je voudrais faire référence à nos travaux (ENSTA et BERTIN). Nous avons exploré l'influence des plaques multiperforées sur l'apparition de la cavitation. Nos plaques étaient munies d'ouvertures fixes, elles n'ont donc pas tout à fait la même conception que votre vanne.

Néanmoins, nous avons remarqué que nos résultats différaient de ceux publiés dans la littérature concernant l'orifice unique et qu'il y avait une influence très importante de la distance entre les orifices, c'est-à-dire que lorsque l'on rapproche les orifices entre eux cela implique leur interaction.

Pour ne pas avoir d'interaction du tout, il faut que les orifices soient très éloignées. D'après ce que l'on a vu sur votre dessin de vanne, cette condition n'est pas remplie, même si vous avez la distance entre les orifices de l'ordre de deux fois et demi le diamètre vous avez une interaction entre les jets, et la cavitation apparaît pour des indices de cavitation deux à trois fois supérieurs.

La théorie explique ceci de la façon suivante: aussi bien les orifices voisins les uns des autres que la paroi solide d'une canalisation peuvent influencer la formation des jets, il y aura des modifications de l'enroulement tourbillonnaire dans les couches de cisaillement ce qui entraine l'apparition de la cavitation dans les jets pour des sigma plus élevés que celui d'un orifice unique de même surface de passage.

M. BONAZZI: Je suis parfaitement d'accord avec ce que vous dites. Je dirai simplement que nous nous sommes attachés au début de cette cavitation et aux cavitations caractérisées qui se développent essentiellement à l'intérieur des orifices. On peut penser que cette cavitation interne à l'orifice n'est pas influencée par l'écoulement aval.

Mme NIENALTOWSKA : Mais grosso modo les valeurs de sigma sont de l'ordre de 1 à 2,5 fois plus grands pour des plaques multiperforées que pour la plaque avec le trou unique.

M. BoNAZZI: On parle de ce qui se passe à l'intérieur de l'orifice. Avant de faire tous ces essais sur le modèle monotrou, on a comparé ce que l'on obtenait sur un modèle monotrou représentatif du MONOVAR de la série diamètre 200 avec un
MONOVAR DN 200 dont nous disposions au laboratoire. On a trouvé une très bonne concordance entre les débuts de cavitation que l'on trouvait sur le monotrou et ceux que l'on constate sur la vanne elle-même.

M. FOURNIER: Nous avons vérifié que l'on obtenait le même comportement hydraulique sur une vanne réelle multitrous que sur un modèle monotrou.

M. CANAVELIS : Je voudrais savoir, lorsque vous parlez de début de réduction de débit, à quelle structure d'écoulement cela correspond par rapport à tout ce que l'on a vu comme extension de poche. Le coefficient de débit du trou, c'est pour une poche qui remplit le trou, qui va au-delà ? Je n'ai pas bien vu comment cela se situe parmi les diffërentes figures.

Deuxième question : Est-ce au niveau des localisations d'érosion éventuelles, s'il y en a, ou est-ce localisé dans la plaque fixe aval, ou même mobile? A mon avis, cela peut remonter.

M. BONAZZI: Le début de réduction de débit correspond à des poches très développées qui se prolongent jusqu'à l'aval de la plaque. C'est pratiquement tout le passage de la vanne qui est en vapeur sur le diamètre de la tuyauterie.

M. Canavelis: Ce que l'on observe, peut-on le considérer comme une cavitation de jet dans la masse ou un décollement sur les bords à l'entrée, ou est-ce une somme des deux ?

M. BoNAZZI : C'est une somme des deux. A $100 \%$ d'ouverture, c'est plutôt une cavitation de jet, et à ouverture partielle, on note un décollement sur les bords des orifices lié à une cavitation du jet induit.

M. CoLAS : Pour les deux plaques, la plaque mobile et la plaque fixe, il y a interaction de l'une sur l'autre, d'après ce que l'on peut en juger par la figure, quand on est en situation de cavitation. Avez-vous fait des tests ou des mesures quelconques qui permettent de voir si l'on n'a pas de mouvement relatif vibratoire entre ces deux plaques.

M. BONAZZI: Je n'ai pas fait ce genre de mesure puisque, sur le modèle, je réglais l'ouverture et, ensuite, je bloquais les deux plaques.

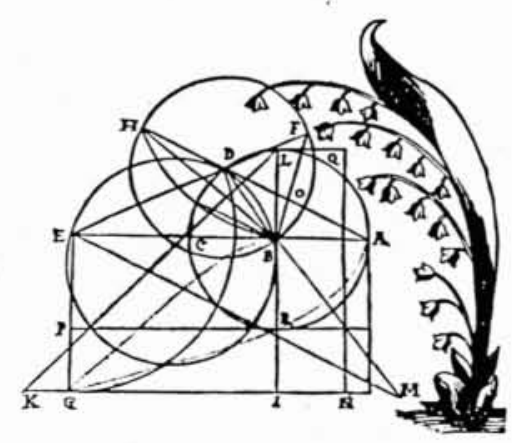

